\title{
A new proof of the Hölder continuity of solutions to $p$-Laplace type parabolic equations.
}

\author{
Ugo Gianazza \\ Dipartimento di Matematica "F. Casorati", Università di Pavia \\ via Ferrata 1, 27100 Pavia, Italy \\ email: gianazza@imati.cnr.it \\ Mikhail Surnachev \\ Department of Mathematics, Swansea University \\ Swansea SA2 8PP, UK \\ email: mamsu@swansea.ac.uk \\ Vincenzo Vespri \\ Dipartimento di Matematica "U. Dini", Università di Firenze \\ viale Morgagni 67/A, 50134 Firenze, Italy \\ email: vespri@math.unifi.it
}

\begin{abstract}
It is a well-known fact that solutions to nonlinear parabolic partial differential equations of $p$-laplacian type are Hölder continuous. One of the main features of the proof, as originally given by DiBenedetto and DiBenedetto-Chen, consists in studying separately two cases, according to the size of the solution. Here we present a new proof of the Hölder continuity of solutions, which is based on the ideas used in the proof of the Harnack inequality for the same kind of equations recently given by E. DiBenedetto, U. Gianazza and V. Vespri. Our method does not rely on any sort of alternative, and has a strong geometric character.
\end{abstract}

AMS Subject Classification (2000): Primary 35K65, 35B65; Secondary 35B45

Key Words: parabolic, degenerate, Harnack, Hölder, intrinsic scaling.

\section{Introduction and Main Result}

Let $\Omega$ be a domain in $\mathbb{R}^{n}$. For $T>0$ let $\Omega_{T}$ denote the cylindrical domain $\Omega \times(0, T]$. In the cylinder $\Omega_{T}$ consider the quasi-linear parabolic differential equation

$$
u_{t}=\operatorname{div} \mathbf{A}(x, t, u, D u) .
$$

The function $\mathbf{A}: \Omega_{T} \times \mathbb{R}^{n+1} \rightarrow \mathbb{R}^{n}$ is a Carathéodory function satisfying

$$
\begin{gathered}
\mathbf{A}(x, t, u, D u) \cdot D u \geq C_{o}|D u|^{p}, \\
|\mathbf{A}(x, t, u, D u)| \leq C_{1}|D u|^{p-1}
\end{gathered}
$$

almost everywhere in $\Omega_{T}$ for $p>2$ and where $C_{o}, C_{1}$ are given positive constants.

A function

$$
u \in C_{l o c}\left(0, T ; L_{l o c}^{2}(\Omega)\right) \cap L_{l o c}^{p}\left(0, T ; W_{l o c}^{1, p}(\Omega)\right)
$$


is a local weak super(sub)solution to (1.1) if for every compact set $K \subset \Omega$ and for every subinterval $\left[t_{1}, t_{2}\right] \subset$ $(0, T]$ one has

$$
\left.\int_{K} u \varphi d x\right|_{t_{1}} ^{t_{2}}+\int_{t_{1}}^{t_{2}} \int_{K}\left[-u \varphi_{t}+\mathbf{A}(x, t, u, D u) \cdot D \varphi\right] d x d t \geq(\leq) 0
$$

for all nonnegative test functions

$$
\varphi \in W_{l o c}^{1,2}\left(0, T ; L^{2}(K)\right) \cap L_{l o c}^{p}\left(0, T ; W_{o}^{1, p}(K)\right) .
$$

We say that $u$ is a local weak solution if it is both a local weak sub- and supersolution.

We say that a constant $\gamma=\gamma($ data $)$ if it can be quatitatively expressed in terms of $n, p, C_{o}, C_{1}$. For $y \in \mathbb{R}^{n}$ and $\rho>0$ let

$$
K_{\rho}^{y}=\left\{x \in \mathbb{R}^{n}:\left|x_{i}-y_{i}\right|<\rho / 2, i=1, \ldots, n\right\} .
$$

For a given cylinder $Q=K_{\rho}^{x} \times\left[t_{o}-\theta \rho^{p}, t_{o}\right]$ we denote $\frac{1}{2} Q=K_{\rho / 2}^{x} \times\left[t_{o}-\theta(\rho / 2)^{p}, t_{o}\right]$. The main result of this paper is the following

Theorem 1.1. Let $u$ be a locally bounded weak solution of (1.1) in $\Omega_{T}$. Then, up to modification on a set of measure zero, $u$ is locally Hölder continuous in $\Omega \times(0, T]$. The Hölder constants can be determined a priori only in terms of the data.

Indeed, that locally bounded weak solutions to (1.1) are locally Hölder continuos is not a new result: the proof of this fact was first given by E. DiBenedetto in [8] for the degenerate case $p>2$, and by Y.Z. Chen and E. DiBenedetto for the singular case $1<p<2$ in [1], [2]. The book [3] contains the proof of the Hölder continuity of solutions for equations with a very general structure. The main ideas underlying the original proof by DiBenedetto, namely the so-called intrisic scaling method, are discussed in [7]. A thorough presentation of this same set of techniques is given in the recent monograph [9].

Here the focus is on the degenerate case, i.e. when $p>2$; the corresponding approach to the Hölder continuity for the singular case, namely when $1<p<2$, will be dealt with in [6].

The structure of the proof given in [8] is based on studying separately two cases. Either one can find a cylinder of the type $K_{\rho}^{x_{o}} \times\left[t_{o}-\theta \rho^{p}, t_{o}\right]$ where $u$ is mostly large, or such a cylinder cannot be found. In either case the conclusion is that the essential oscillation of $u$ in a smaller cylinder about $\left(x_{o}, t_{o}\right)$ decreases in a way that can be quantitatively measured.

The actual technical implementation of the previous alternative is not an easy job; the point in giving a new proof of the by-now classical result by DiBenedetto is to show how a certain set of ideas, which led to the proof of the Harnack inequality in [5], can simplify the argument, and avoid any use of alternatives. We believe that the new proof has a further significant feature, namely its strong geometric character.

Three final comments are due here:

- In order to present the essence of the approach, only the case of homogeneous-structure equations is dealt with here, but with little further effort the full quasi-linear case could be considered too.

- In the following we deal with equations of $p$-laplacian type, but, with some care, the same kind of arguments can be used to prove the Hölder continuity of quasi-linear parabolic differential equation of the sort

$$
u_{t}=\operatorname{div} \mathbf{A}(x, t, u, D u),
$$

where the function $\mathbf{A}: \Omega_{T} \times \mathbb{R}^{n+1} \rightarrow \mathbb{R}^{n}$ is a Carathéodory function satisfying the structure conditions

$$
\begin{gathered}
\mathbf{A}(x, t, u, D u) \cdot D u \geq C_{o}|u|^{m-1}|D u|^{2} \\
|\mathbf{A}(x, t, u, D u)| \leq C_{1}|u|^{m-1}|D u|
\end{gathered}
$$

almost everywhere in $\Omega_{T}$ for $m>1, C_{o}$ and $C_{1}$ being given positive constants. The prototype of this kind of equations is the so-called porous medium equation, namely

$$
u_{t}-\Delta|u|^{m-1} u=0,
$$


which has been extensively studied in the last thirty years, in the context of non-linear diffusion phenomena. For a thorough treatment of this very interesting topic, see for example [10]. The care we were referring above, is due to the fact that if $u$ is a solution to a porous medium equation, given a generic constant $c \neq 0$, in general $u+c$ is not a solution. Therefore one has to take into account that solutions are signed solutions, and this brings about some further technical difficulties.

- In the rest of the paper by solutions we will always mean weak solutions.

Acknowledgement: This paper was written during the INdAM Intensive Period Geometric Properties of Nonlinear Local and Non local Problems. The authors thank INdAM for the support that made the event possible.

\section{Main Lemma and Proof of Theorem 1.1}

As it will be clear at the end of this Section, the proof of Theorem 1.1 is a straightforward consequence of the following lemma.

Lemma 2.1. Let $u$ be a nonnegative solution to (1.1) in the cylinder $Q=K_{4}^{0} \times(-2, T]$. There exist constants $0<\gamma_{1}<\gamma_{2}$ and $\mu>0$, depending only on $C_{o}, C_{1}, n, p$, such that if $T \geq \gamma_{2}$ and

$$
\left|\left\{(x, t) \in K_{1}^{0} \times(-1,0]: u(x, t) \geq \frac{1}{2}\right\}\right| \geq \frac{1}{2}
$$

then

$$
\underset{Q^{\prime}}{\operatorname{essinf}} u \geq \mu, \quad \text { where } Q^{\prime}=K_{1}^{0} \times\left(\gamma_{1}, \gamma_{2}\right] .
$$

The proof of this lemma is the most technically involved part of the paper and we postpone its proof to the last Section of the paper. Let

$$
\gamma_{3}=\gamma_{2}-\gamma_{1}, \quad \gamma_{4}=2+\gamma_{2} .
$$

It is obvious that $0<\gamma_{3}<\gamma_{4}$. The following proposition is elementary and we skip its proof.

Proposition 2.1. Let $u$ be a solution to (1.1) in the cylinder $Q=K_{4 \rho}^{x_{o}} \times\left[t_{o}-\gamma_{4} \omega^{2-p} \rho^{p}, t_{o}\right]$, where $\omega>0$. Let $\beta \in \mathbb{R}$. Set

$$
z= \pm \frac{u+\beta}{\omega}, \quad x=x_{o}+\rho y, \quad t=t_{o}+\left(\tau-\gamma_{2}\right) \omega^{2-p} \rho^{p} ;
$$

then $z$ is a solution to the equation

$$
z_{\tau}=\operatorname{div} \mathbf{A}_{1}(y, \tau, z, D z)
$$

in the cylinder $Q_{1}=K_{4}^{0} \times\left[-2, \gamma_{2}\right]$, where $\mathbf{A}_{1}$ is a Carathéodory function satisfying

$$
\begin{gathered}
\mathbf{A}_{1}(y, \tau, z, D z) \cdot D z \geq C_{o}|D z|^{p}, \\
\left|\mathbf{A}_{1}(y, \tau, z, D z)\right| \leq C_{1}|D z|^{p-1}
\end{gathered}
$$

The following corollary of Lemma 2.1 is a classical step in the proof of the Hölder continuity of solutions to degenerate and singular parabolic partial differential equations.

Lemma 2.2. Let $u$ be a solution to (1.1) in the cylinder $Q=K_{4 \rho}^{x_{o}} \times\left[t_{o}-\gamma_{4} \omega^{2-p} \rho^{p}, t_{o}\right]$, where $\omega>0$. Let $\operatorname{ess}^{\operatorname{osc}_{Q}} u \geq \omega$. Let $Q^{\prime}=K_{\rho}^{x_{o}} \times\left[t_{o}-\gamma_{3} \omega^{2-p} \rho^{p}, t_{o}\right]$. Then

$$
\underset{Q^{\prime}}{\operatorname{ess} \operatorname{osc}} u \leq \underset{Q}{\operatorname{essosc}} u-\mu \omega,
$$

where $\mu$ is the quantity given by Lemma 2.1 
Proof. Let

$$
m=\underset{Q}{\operatorname{essinf}} u, \quad M=\underset{Q}{\operatorname{ess} \sup } u, \quad \xi=\frac{M-m}{\omega} \geq 1,
$$

and

$$
z=\frac{u-m}{\omega}, \quad x=x_{o}+\rho y, \quad t=t_{o}+\left(\tau-\gamma_{2}\right) \omega^{2-p} \rho^{p} .
$$

By Proposition 2.1, $z$ is a non-negative solution to

$$
z_{\tau}=\operatorname{div} \mathbf{A}_{1}(y, \tau, z, D z)
$$

in the cylinder $Q_{1}=K_{4}^{0} \times\left[-2, \gamma_{2}\right]$, and the vector field $\mathbf{A}_{1}$ satisfies the same structural conditions as $\mathbf{A}$. One of the following statements holds:

$$
\begin{aligned}
& \left|\left\{z \geq \frac{\xi}{2}\right\} \cap K_{1}^{0} \times[-1,0]\right| \geq \frac{1}{2}, \\
& \left|\left\{z \geq \frac{\xi}{2}\right\} \cap K_{1}^{0} \times[-1,0]\right|<\frac{1}{2} .
\end{aligned}
$$

Let (2.1) hold: then $\left|\left\{z \geq \frac{1}{2}\right\} \cap K_{1}^{0} \times[-1,0]\right| \geq \frac{1}{2}$, and by Lemma 2.1 we obtain

$$
\left.\left.z \geq \mu \quad \text { a.e. in } \quad K_{1}^{0} \times\right] \gamma_{1}, \gamma_{2}\right] .
$$

Hence

$$
\underset{Q^{\prime}}{\operatorname{ess} \inf } u \geq m+\mu \omega .
$$

and the assertion of the lemma easily follows.

Analogously, if (2.2) holds, then the function

$$
\hat{z}=\xi-z=\frac{M-u}{\omega}
$$

satisfies

$$
\hat{z} \geq \mu \quad \text { a.e. in } K_{1}^{0} \times\left[\gamma_{1}, \gamma_{2}\right]
$$

and

$$
\underset{Q^{\prime}}{\operatorname{ess} \sup } u \leq M-\mu \omega .
$$

The proof of the following energy inequalities can be found in [3].

Proposition 2.2. Let the cylinder $Q=K_{\rho}^{y} \times\left[t_{1}, t_{2}\right] \subset \Omega_{T}$ and $\xi$ be a non-negative piecewise-smooth test function vanishing on the lateral boundary of $Q$. If $u$ is a subsolution to the equation (1.1) in $\Omega_{T}$, then for any $k \in \mathbb{R}$ we have

$$
\begin{gathered}
\left.\int_{K_{\rho}^{y}}(u-k)_{+}^{2} \xi^{p} d x\right|_{t_{1}} ^{t_{2}}+C_{o} \iint_{Q}\left|D(u-k)_{+}\right|^{p} \xi^{p} d x d t \\
\leq p \iint_{Q}(u-k)_{+}^{2} \xi^{p-1} \xi_{t} d x d t+\tilde{\gamma}_{o} \iint_{Q}(u-k)_{+}^{p}|D \xi|^{p} d x d t .
\end{gathered}
$$


Proposition 2.3. Let the cylinder $Q=K_{\rho}^{y} \times\left[t_{1}, t_{2}\right] \subset \Omega_{T}$ and $\xi$ be a non-negative piecewise-smooth test function vanishing on the lateral boundary of $Q$. If $u$ is a supersolution to (1.1) in $\Omega_{T}$, then for any $k \in \mathbb{R}$ we have

$$
\begin{gathered}
\left.\int_{K_{\rho}^{y}}(u-k)_{-}^{2} \xi^{p} d x\right|_{t_{1}} ^{t_{2}}+C_{o} \iint_{Q}\left|D(u-k)_{-}\right|^{p} \xi^{p} d x d t \\
\leq p \iint_{Q}(u-k)_{-}^{2} \xi^{p-1} \xi_{t} d x d t+\tilde{\gamma}_{o} \iint_{Q}(u-k)_{-}^{p}|D \xi|^{p} d x d t .
\end{gathered}
$$

Remark 2.1. The proof shows that in both cases

$$
\tilde{\gamma}_{o}=\left(2 C_{1}\right)^{p}\left(\frac{p-1}{C_{o}}\right)^{p-1} .
$$

Assuming for the moment the validity of Lemma 2.1, we proceed with the

Proof of Theorem 1.1. Let $\left(x_{o}, t_{o}\right) \in \Omega_{T}$ and set

$$
d_{x}=\operatorname{dist}\left(x_{o}, \partial \Omega\right), \quad d_{t}=t_{o}>0 .
$$

Assume that $\sup _{\Omega_{T}}|u|=M<\infty$. Let $\left\{\omega_{j}\right\}_{j=0}^{\infty},\left\{\rho_{j}\right\}_{j=0}^{\infty}$ be the sequences of positive numbers defined by

$$
\rho_{j}=\varepsilon \rho_{j-1}, \quad \omega_{j}=\delta \omega_{j-1},
$$

where $\delta \in(0,1), \varepsilon \in\left(0, \frac{1}{4}\right], \varepsilon<\delta$ are to be chosen. Notice that the condition $\varepsilon<\delta$ guarantees that the sequence of cylinders shrinks to a point. We also require that $\rho_{o} \leq d_{x}$ and $\gamma_{4} \omega_{o}^{2-p}\left(\frac{\rho_{o}}{4}\right)^{p} \leq d_{t}$. Let

$$
Q_{j}=K_{\rho_{j}}^{x_{o}} \times\left[t_{o}-\gamma_{4} \omega_{j}^{2-p}\left(\frac{\rho_{j}}{4}\right)^{p}, t_{o}\right]
$$

and denote

$$
A_{j}=\underset{Q_{j}}{\operatorname{ess} \operatorname{osc} u}
$$

We want to show that there exists a constant $\Lambda=\Lambda($ data $)>1$, such that

$$
A_{j} \leq \Lambda \omega_{j} \quad \Rightarrow \quad A_{j+1} \leq \Lambda \omega_{j+1} .
$$

Suppose that $\varepsilon$ and $\delta$ are such that $\gamma_{4} \varepsilon^{p} \delta^{2-p} \leq \gamma_{3}$.

Assume first that $A_{j} \geq \omega_{j}$. Then, in virtue of Lemma 2.2,

$$
\begin{gathered}
A_{j+1} \leq A_{j}-\mu \omega_{j} \leq(\Lambda-\mu) \omega_{j}=\frac{\Lambda-\mu}{\delta \Lambda} \Lambda \omega_{j+1} \\
\leq \Lambda \omega_{j+1} \quad \text { if } \quad \Lambda-\mu \leq \delta \Lambda \Leftrightarrow \Lambda \leq \frac{\mu}{1-\delta} .
\end{gathered}
$$

On the other hand, if $A_{j} \leq \omega_{j}$, then

$$
A_{j+1} \leq A_{j} \leq \omega_{j}=\frac{1}{\delta} \omega_{j+1} \leq \Lambda \omega_{j+1} \quad \text { if } \quad \frac{1}{\delta} \leq \Lambda .
$$

Hence, any $\Lambda$ such that

$$
\frac{1}{\delta} \leq \Lambda \leq \frac{\mu}{1-\delta} .
$$


will do. It is clear that the previous inequality is satisfied if

$$
\frac{1}{\delta} \leq \frac{\mu}{1-\delta} \quad \Leftrightarrow \quad \delta \geq \frac{1}{1+\mu}
$$

Take $\delta=\frac{1}{1+\mu}$ and $\Lambda=1+\mu$. Set

$$
\varepsilon=\min \left\{\frac{1}{4},\left(\frac{\gamma_{3}}{\gamma_{4}} \delta^{p-2}\right)^{\frac{1}{p}}\right\}, \quad \omega_{o}=2 M, \quad \text { and } \quad \rho_{o}=\min \left\{d_{x}, 4\left(\frac{d_{t}}{\gamma_{4} \omega_{o}^{2-p}}\right)^{\frac{1}{p}}\right\} .
$$

Then it is immediate to see that $\underset{Q_{o}}{\operatorname{ess} \operatorname{osc}} u \leq(1+\mu) \omega_{o}$, which implies

$$
\underset{Q_{j}}{\operatorname{ess} \operatorname{osc}} u \leq(1+\mu) \omega_{j}=(1+\mu)^{1-j} \omega_{o} .
$$

Let

$$
Q_{r, s}^{x_{o}, t_{o}}=K_{r}^{x_{o}} \times\left(t_{o}-s, t_{o}\right] \subset \Omega_{T}
$$

and

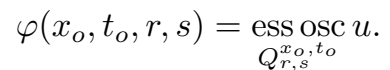

Choosing $j$ in such a way that $Q_{r, s}^{x_{o}, t_{o}} \subset Q_{j}$, we have

$$
\varphi\left(x_{o}, t_{o}, r, s\right) \leq(1+\mu)^{2} \omega_{o} \max \left\{\left(\frac{s}{\gamma_{4}\left(\rho_{o} / 4\right)^{p} \omega_{o}^{2-p}}\right)^{\alpha_{1}},\left(\frac{r}{\rho_{o}}\right)^{\alpha_{2}}\right\},
$$

with

$$
\alpha_{1}=\frac{1}{\log _{1+\mu} \frac{1}{\varepsilon^{p} \delta^{2-p}}}, \quad \alpha_{2}=\frac{1}{\log _{1+\mu} \frac{1}{\varepsilon}} .
$$

Since $\varepsilon^{p} \delta^{2-p} \leq \frac{\gamma_{3}}{\gamma_{4}}<1$, and by possibly reducing $\mu$ and enlarging $\gamma_{1}$, we have that both $\alpha_{1}, \alpha_{2} \in(0,1)$. Notice that

$$
\frac{\alpha_{1}}{\alpha_{2}}=\frac{1}{\log _{\varepsilon} \varepsilon^{p} \delta^{2-p}}=\frac{1}{p+(2-p) \log _{\varepsilon} \delta}>\frac{1}{p} .
$$

The rest of the proof follows in a standard way.

\section{Auxiliary Propositions and Technical Results}

In the following we gather various technical results, which are used in the proof of Lemma 2.1. Some of the statements will be given without proofs (and in such a case we refer the reader to [5] or [3]); others will be explicitely proved, even if the arguments are mainly proper modifications of analogous results given in [5].

The first two lemmata, which we state for sub- and supersolutions separately, are one of the traditional and most widely used tools in the regularity theory.

Lemma 3.1. Let $u$ be a subsolution to (1.1) in the cylinder $Q=K_{2 \rho}^{y} \times\left[t_{1}-\theta(2 \rho)^{p}, t_{1}\right]$. Let $\mu_{+} \geq$ $\operatorname{esssup}_{Q} u(x, t)$. Then for any $\omega>0$ and $a \in(0,1)$ there exists a number $s$, which depends only on the data, $a$, and $\theta \omega^{p-2}$, such that if

$$
\left|\left\{(x, t) \in Q: u(x, t)>\mu_{+}-\omega\right\}\right| \leq s|Q|,
$$

then we have

$$
\underset{\frac{1}{2} Q}{\operatorname{ess} \sup } u(x, t) \leq \mu_{+}-a \omega .
$$


Lemma 3.2. Let $u$ be a supersolution to (1.1) in the cylinder $Q=K_{2 \rho}^{y} \times\left[t_{1}-\theta(2 \rho)^{p}, t_{1}\right]$. Let $\mu_{-} \leq$ $\operatorname{essinf}_{Q} u(x, t)$. Then for any $\omega>0$ and $a \in(0,1)$ there exist a number $s$, which depends only on the data, $a$, and $\theta \omega^{p-2}$, such that if

$$
\left|\left\{(x, t) \in Q: u(x, t)<\mu_{-}+\omega\right\}\right| \leq s|Q|
$$

then we have

$$
\underset{\frac{1}{2} Q}{\operatorname{essinf}} u(x, t) \geq \mu_{-}+a \omega .
$$

The proof shows that the value of $s$ in Lemmas 3.1 and 3.2 is the same for the same values of $a$ and $\theta \omega^{p-2}$, namely

$$
s=\left(\frac{1-a}{\gamma(\text { data })}\right)^{n+p} \frac{\left[\theta \omega^{p-2}\right]^{n / p}}{\left[1+\theta \omega^{p-2}\right]^{(n+p) / p}} .
$$

Given $a$ and $\xi=\theta \omega^{p-2}$ we denote the corresponding value of $s$ by $s(a, \xi)$.

The next lemma is a variant of the previous result.

Lemma 3.3. Let $u$ be a non-negative supersolution to (1.1) in the cylinder $Q=K_{2 \rho}^{y} \times\left[t_{1}-\theta(2 \rho)^{p}, t_{1}\right]$. Suppose that $\operatorname{ess}_{\inf _{K_{2 \rho}^{y}}} u\left(x, t_{1}-\theta(2 \rho)^{p}\right) \geq k$. Then there exists $\nu=\nu($ data $)>0$ such that if $\theta<\nu k^{2-p}$,

$$
\underset{K_{\rho}^{y}}{\operatorname{ess} \inf } u\left(x, t_{1}\right) \geq k / 2
$$

A consequence of the previous lemma is

Corollary 3.1. Let $u$ be a non-negative supersolution to (1.1) in the cylinder $K_{2 \rho}^{y} \times\left[t_{1}, t_{1}+T\right]$. Let $\operatorname{essinf}_{K_{2 \rho}^{y}} u\left(x, t_{1}\right) \geq k$. Then for all $t \in\left(t_{1}, t_{1}+T\right]$ we have

$$
\underset{K_{\rho}^{y}}{\operatorname{ess}} \inf u(x, t) \geq \frac{k}{2}\left(1+\frac{t-t_{1}}{\nu k^{2-p}(2 \rho)^{p}}\right)^{\frac{1}{2-p}},
$$

where $\nu$ is the constant from the statement of Lemma 3.3.

Proof. It is clear, that for any $\tau \in[0,1]$ we have

$$
\underset{K_{2 \rho}^{y}}{\operatorname{ess} \inf } u\left(x, t_{1}\right) \geq \tau k .
$$

If $t-t_{1} \leq \nu k^{2-p}(2 \rho)^{p}$, then Lemma 3.3 yields $u(x, t) \geq k / 2$ a.e. in $K_{\rho}^{y}$. Now assume that $t-t_{1}>\nu k^{2-p}(2 \rho)^{p}$. In (3.2) take

$$
\tau=\left(\frac{\nu k^{2-p}(2 \rho)^{p}}{t-t_{1}}\right)^{\frac{1}{p-2}}
$$

and apply Lemma 3.3 with $k$ replaced by $\tau k$. This gives

$$
u(x, t) \geq\left(\frac{\nu k^{2-p}(2 \rho)^{p}}{t-t_{1}}\right)^{\frac{1}{p-2}} \frac{k}{2} \quad \text { a.e. in } K_{\rho}^{y} .
$$

The combination of the estimates for $t \leq t_{1}+\nu k^{2-p}(2 \rho)^{p}$ and $t>t_{1}+\nu k^{2-p}(2 \rho)^{p}$ concludes the proof.

The next lemma is analogous to Proposition 6.1 of [5].

Lemma 3.4. Let $v$ be a non-negative supersolution of (1.1) in the cylinder $Q=K_{4}^{0} \times[0, T]$. Assume we have

$$
\left|\left\{x \in K_{2}^{0}: v(x, t) \geq 1\right\}\right| \geq \alpha\left|K_{2}^{0}\right|,
$$


for all $t \in[0, T]$, where $\alpha \in(0,1)$ is a given constant. Then for any $\varepsilon>0$ there exist $\theta=\theta(\alpha, \varepsilon$, data $)>0$ such that if $T \geq \theta 2^{p+1}$, then for the cylinder $Q_{1}=K_{2}^{0} \times\left[\theta 2^{p}, \theta 2^{p+1}\right] \subset Q$, we have

$$
\left|\left\{(x, t) \in Q_{1}: v(x, t)<\theta^{\frac{1}{2-p}}\right\}\right| \leq \varepsilon\left|Q_{1}\right| .
$$

Moreover, $\theta$ is a monotone decreasing function of $\varepsilon$. Given $\alpha$ and $\varepsilon$ we denote the corresponding $\theta$ by $\theta(\varepsilon, \alpha)$.

Proof. Denote $k_{j}=2^{-j}$ for $j=0,1, \ldots, j_{*}$, where $j_{*}$ will be chosen later, and let $Q_{2}=K_{4}^{0} \times\left[0,2^{p+1} \theta\right]$, where the constant $\theta$ will be specified later. Take the piecewise-smooth cut-off function $\xi(x, t)$ such that $\xi=1$ on $Q_{1}, 0 \leq \xi \leq 1$ on $Q_{2}, \xi$ vanishes on the parabolic boundary of $Q_{2},\left|\xi_{t}\right| \leq \frac{2}{2^{p} \theta}$ and $|D \xi| \leq 1$.

From inequality (2.4), we obtain

$$
\iint_{Q_{1}}\left|D\left(v-k_{j}\right)_{-}\right|^{p} d x d t \leq \gamma\left|Q_{2}\right|\left(\frac{k_{j}^{2}}{\theta}+k_{j}^{p}\right) .
$$

Take $\theta=k_{j_{*}}^{2-p}$. Then the last inequality yields

$$
\iint_{Q_{1}}\left|D\left(v-k_{j}\right)_{-}\right|^{p} d x d t \leq \gamma\left|Q_{1}\right| k_{j}^{p}
$$

Denote

$$
A_{j}=\left\{(x, t) \in Q_{1}: v(x, t)<k_{j}\right\} \quad \text { and } \quad A_{j}(\tau)=\left\{x \in K_{2}^{0}: v(x, \tau)<k_{j}\right\} .
$$

Using De Giorgi - Poincaré inequality (see [3], Chapter I, Lemma 2.2), thanks to the hypotheses we obtain

$$
\begin{gathered}
\left(k_{j}-k_{j+1}\right)\left|A_{j+1}(\tau)\right| \leq \frac{\gamma(n)}{\left|K_{2}^{0} \backslash A_{j}(\tau)\right|} \int_{A_{j}(\tau) \backslash A_{j+1}(\tau)}\left|D\left(v-k_{j}\right)_{-}\right| d x \\
\leq \frac{\gamma(n)}{\alpha\left|K_{2}^{0}\right|} \int_{A_{j}(\tau) \backslash A_{j+1}(\tau)}\left|D\left(v-k_{j}\right)_{-}\right| d x .
\end{gathered}
$$

Integration of the last inequality over $\tau \in\left[2^{p} \theta, 2^{p+1} \theta\right]$ yields

$$
\begin{gathered}
\frac{k_{j}}{2}\left|A_{j+1}\right| \leq \frac{\gamma}{\alpha} \iint_{A_{j} \backslash A_{j+1}}\left|D\left(v-k_{j}\right)_{-}\right| d x d t \\
\leq \frac{\gamma}{\alpha}\left(\iint_{A_{j} \backslash A_{j+1}}\left|D\left(v-k_{j}\right)_{-}\right|^{p} d x d t\right)^{\frac{1}{p}}\left|A_{j} \backslash A_{j+1}\right|^{\frac{p-1}{p}} \\
\leq \frac{\gamma}{\alpha} k_{j}\left|Q_{1}\right|^{\frac{1}{p}}\left|A_{j} \backslash A_{j+1}\right|^{\frac{p-1}{p}} .
\end{gathered}
$$

Hence,

$$
\left|A_{j+1}\right|^{\frac{p}{p-1}} \leq\left(\frac{\gamma}{\alpha}\right)^{\frac{p}{p-1}}\left|Q_{1}\right|^{\frac{1}{p-1}}\left|A_{j} \backslash A_{j+1}\right| .
$$

Summing the last inequality over $j=0,1, \ldots, j_{*}-1$, we obtain

$$
j_{*}\left|A_{j_{*}}\right|^{\frac{p}{p-1}} \leq\left(\frac{\gamma}{\alpha}\right)^{\frac{p}{p-1}}\left|Q_{1}\right|^{\frac{p}{p-1}}
$$

whence

$$
\left|A_{j_{*}}\right| \leq \frac{\gamma}{\alpha}\left(\frac{1}{j_{*}}\right)^{\frac{p-1}{p}}\left|Q_{1}\right|
$$

The lemma is proved with

$$
j_{*}=\left(\frac{\gamma}{\varepsilon \alpha}\right)^{\frac{p}{p-1}}, \quad \theta(\varepsilon, \alpha)=2^{(p-2) j_{*}} .
$$

Now we prove the following 
Proposition 3.1. Let $v$ be as in Lemma 3.4. Denote

$$
Q(\theta)=K_{1}^{0} \times\left[\left(2^{p+1}-1\right) \theta, 2^{p+1} \theta\right] .
$$

There exists a constant $\theta_{o}=\theta_{o}($ data, $\alpha)$ such that

$$
\underset{Q(\theta)}{\operatorname{essinf}} v(x, t) \geq \frac{1}{2} \theta^{\frac{1}{2-p}}
$$

for all $\theta \geq \theta_{o}$. Given $\alpha$, we denote the corresponding value of $\theta_{o}$ by $\theta_{o}(\alpha)$.

Proof. With reference to Lemma 3.2, let $\varepsilon_{o}=s\left(\frac{1}{2}, 1\right)$. Correspondingly let $\theta_{o}=\theta\left(\alpha, \varepsilon_{o}\right)$ as given by Lemma 3.4. Now choose $\varepsilon<\varepsilon_{o}$ and let $\theta=\theta(\varepsilon, \alpha)$. Then in the cylinder $Q_{1}(\theta)=K_{2}^{0} \times\left[2^{p} \theta, 2^{p+1} \theta\right]$ we have

$$
\left|\left\{(x, t) \in Q_{1}(\theta): v(x, t)<\theta^{\frac{1}{2-p}}\right\}\right|<\varepsilon\left|Q_{1}(\theta)\right| .
$$

In the cylinder $Q_{1}(\theta)$ apply Lemma 3.2 with $\mu_{-}=0$ and $a=\frac{1}{2}$ to conclude the proof.

Proposition 3.2. Let $u$ be a non-negative supersolution to (1.1) in the cylinder $Q=K_{1}^{0} \times[-1,0]$. Let

$$
\left|\left\{u \geq \frac{1}{2}\right\} \cap Q\right| \geq \frac{1}{2}
$$

and

$$
\iint_{Q}\left|D\left(u-\frac{1}{2}\right)_{-}\right| d x d t \leq \tilde{\gamma}
$$

Then for any $\sigma \in(0,1)$ there exist $\eta_{o}=\eta_{o}($ data, $\sigma, \gamma) \in(0,1)$ and $(y, s) \in Q$ such that

$$
Q_{\sigma} \equiv K_{\eta_{o}}^{y} \times\left[s-\eta_{o}^{p}\left(\frac{1}{4}\right)^{2-p}, s\right] \subset Q
$$

and

$$
\left|\left\{u \geq \frac{1}{4}\right\} \cap Q_{\sigma}\right| \geq \sigma\left|Q_{\sigma}\right| .
$$

Proof. First, we show that there exists $\tau_{*} \in\left[-1,-\frac{1}{16}\right]$ such that

$$
\int_{K_{1}^{0}}\left|D\left(u-\frac{1}{2}\right)-\right|\left(y, \tau_{*}\right) d y \leq 16 \tilde{\gamma}
$$

and

$$
\left|\left\{u\left(y, \tau_{*}\right) \geq \frac{1}{2}\right\} \cap K_{1}^{0}\right| \geq \frac{3}{8} .
$$

It is obvious that the measure of the subset of $[-1,0]$ where (3.3) does not hold does not exceed $\frac{1}{16}$. Consequently, (3.3) holds on a set of measure at least $\frac{15}{16}$. Next, it is easy to see that the set of $\tau \in(-1,0]$ where (3.4) does not hold has measure less than $\frac{14}{16}$. Hence, both (3.3) and (3.4) hold on a set of measure strictly larger than $\frac{1}{16}$.

We apply the result of $[4]$ to $u\left(\cdot, \tau_{*}\right)$ in $K_{1}^{0}$, and deduce that, for any $\bar{\sigma} \in(0,1)$ there exist $y_{1} \in K_{1}^{0}$ and $\bar{\eta} \in(0,1)$ such that

Let $\theta=4^{2-p}$, and set

$$
\left|\left\{x \in K_{\bar{\eta}}^{y_{1}}: u\left(x, \tau_{*}\right)>\frac{3}{8}\right\}\right|>\bar{\sigma}\left|K_{\bar{\eta}}^{y_{1}}\right| .
$$

$$
\tau_{*}^{\prime}=\tau_{*}+\left(\frac{1}{4}\right)^{2-p}\left(\frac{\bar{\eta}}{2}\right)^{p}(1-\bar{\sigma})
$$


Consider the cylinder

$$
Q_{1}=K_{\bar{\eta}}^{y_{1}} \times\left[\tau_{*}, \tau_{*}^{\prime}\right]
$$

Writing the energy inequality (2.4) over the cylinder $Q_{1}$ with $k=\frac{3}{8}$ and a proper cut-off function, we obtain

$$
\begin{gathered}
\max _{\tau_{*} \leq t \leq \tau_{*}^{\prime}} \int_{K_{\bar{\eta} / 2}^{y_{1}}}\left(u-\frac{3}{8}\right)_{-}^{2} d x \leq \gamma\left((1-\bar{\sigma})\left(\frac{3}{8}\right)^{2}\left|K_{\bar{\eta}}^{y_{1}}\right|\right. \\
\left.+\left(\frac{4}{\bar{\eta}}\right)^{p}\left(\frac{3}{8}\right)^{p}\left|K_{\bar{\eta}}^{y_{1}}\right|\left(\frac{1}{4}\right)^{2-p}\left(\frac{\bar{\eta}}{2}\right)^{p}(1-\bar{\sigma})\right) .
\end{gathered}
$$

Therefore, $\forall t \in\left[\tau_{*}, \tau_{*}^{\prime}\right]$

$$
\left|\left\{x \in K_{\bar{\eta} / 2}^{y_{1}}: u(x, t) \leq \frac{1}{4}\right\}\right| \leq 64 \gamma_{1}(1-\bar{\sigma})\left|K_{\bar{\eta} / 2}^{y_{1}}\right|
$$

If we take $\bar{\sigma}=1-\frac{\sigma 2^{-p}}{64 \gamma_{1}}$, then in the cylinder $Q_{2}=K_{\bar{\eta} / 2}^{y_{1}} \times\left[\tau_{*}, \tau_{*}^{\prime}\right]$ we obtain

$$
\left|\left\{u \leq \frac{1}{4}\right\} \cap Q_{2}\right|<\sigma 2^{-p}\left|Q_{2}\right|
$$

Up to a zero measure set, decompose the base of $Q_{2}$ into the $2^{l n}$ congruent cubes $K_{2^{-l-1} \bar{\eta}}^{z_{j}}, j=1, \ldots, 2^{l n}$. Choose the smallest natural number $l$ such that

$$
\left(2^{-l-1} \bar{\eta}\right)^{p} \leq\left(\frac{\bar{\eta}}{2}\right)^{p}(1-\bar{\sigma})
$$

There exists at least $j$ such that in the cylinder $\tilde{Q}_{j}=K_{2^{-l-1} \bar{\eta}}^{z_{j}} \times\left(\tau_{*}, \tau_{*}^{\prime}\right]$ we have

$$
\left|\left\{u \leq \frac{1}{4}\right\} \cap \tilde{Q}_{j}\right|<\sigma 2^{-p}\left|\tilde{Q}_{j}\right|
$$

In the cylinder

$$
Q_{\sigma}=K_{2^{-l-1} \bar{\eta}}^{z_{j}} \times\left[\tau_{*}, \tau_{*}+\left(2^{-l-1} \bar{\eta}\right)^{p}\left(\frac{1}{4}\right)^{2-p}\right]
$$

we have

$$
\left|\left\{u \leq \frac{1}{4}\right\} \cap Q_{\sigma}\right|<\sigma\left|Q_{\sigma}\right|
$$

We will use the following corollary

Corollary 3.2. Let $u$ be as in Proposition 3.2. Then there exist a number $\eta_{o}>0$ and a cylinder

$$
Q_{3}=K_{\eta_{o}}^{y_{o}} \times\left[s_{o}-\left(\frac{\eta_{o}}{2}\right)^{p}\left(\frac{1}{4}\right)^{2-p}, s_{o}\right] \subset K_{1}^{0} \times[-1,0]
$$

such that

$$
\underset{Q_{3}}{\operatorname{essinf}} u \geq \frac{1}{8}
$$

Proof. Take $\sigma=s\left(\frac{1}{2}, 1\right)$ and apply Lemma 3.2 in the cylinder $Q_{\sigma}$ constructed in Proposition 3.2 with $\mu_{-}=0, \omega=\frac{1}{4}, a=\frac{1}{2}$. 


\section{Proof of Lemma 2.1}

First, from the energy estimate (2.4) it follows that

$$
\iint_{K_{1}^{0} \times[-1,0]}\left|D\left(u-\frac{1}{2}\right)_{-}\right| d x d t \leq \gamma(\text { data }) .
$$

Next, use Corollaries 3.2 and 3.1 to obtain $y_{o} \in K_{1}^{0}, \eta_{o} \in(0,1)$ and $t_{o} \in\left[-\frac{1}{16}, 0\right]$ such that

$$
\underset{\substack{K_{\eta_{o} / 2}^{y_{o}} \\ \operatorname{essinf}}}{\sin }(x, t) \geq \frac{1}{16}\left(1+\frac{t-t_{o}}{\nu 8^{p-2} \eta_{o}^{p}}\right)^{\frac{1}{2-p}}, \quad t \geq t_{o},
$$

where $\eta_{o}=\eta_{o}($ data $)>0$. Hence,

$$
\underset{\substack{K_{\eta_{o} / 2}^{y_{o}} \\ \operatorname{essinf}}}{\sin }(x, 0) \geq \mu_{o}:=\frac{1}{16}\left(1+\frac{1}{\nu 8^{p-2} \eta_{o}^{p}}\right)^{\frac{1}{2-p}} .
$$

Apply Corollary 3.1 again to obtain that

$$
\underset{\substack{K_{\eta_{o} / 4}^{y_{o}} \\ \operatorname{essinf}}}{\operatorname{yon}}(x, t) \geq \psi(t):=\frac{\mu_{o}}{2}\left(1+\frac{t}{\nu \mu_{o}^{2-p}\left(\eta_{o} / 2\right)^{p}}\right)^{\frac{1}{2-p}}, \quad t \geq 0 .
$$

Change the variables in equation (1.1) as

$$
u(x, t)=v(x, t) \psi(t), \quad t=t(\tau),
$$

where $\tau$ is a solution to the problem

$$
\frac{d \tau}{d t}=\psi^{p-2}(t), \quad \tau(0)=0
$$

One can see that

$$
\begin{gathered}
\tau(t)=\frac{\nu}{2^{p-2}}\left(\frac{\eta_{o}}{2}\right)^{p} \ln \left(1+\frac{t}{\nu \mu_{o}^{2-p}\left(\eta_{o} / 2\right)^{p}}\right), \\
\psi(t(\tau))=\frac{\mu_{o}}{2} \exp \left[\frac{2^{p-2} \tau}{(2-p) \nu\left(\eta_{o} / 2\right)^{p}}\right]
\end{gathered}
$$

and for all $\tau \geq 0$ we have

$$
\underset{K_{\eta_{o} / 4}^{y_{o}}}{\operatorname{ess} \inf } v(x, \tau) \geq 1
$$

It can be verified that $v$ is a supersolution to the equation

$$
v_{\tau}=\operatorname{div} \mathbf{A}_{1}(x, \tau, v, D v)
$$

where

$$
\mathbf{A}_{1}(x, \tau, v, D v)=\psi^{1-p}(t) \mathbf{A}(x, t, \psi v, \psi D v) .
$$

Moreover, $\mathbf{A}_{1}$ satisfies the same structural conditions as A. Applying Proposition 3.1 we obtain that

$$
\underset{K_{1}^{0}}{\operatorname{ess} \inf } v(x, \tau) \geq \frac{1}{2} \theta^{\frac{1}{2-p}}
$$

with $\theta=\theta_{o}\left(\left(\frac{\eta_{o}}{4}\right)^{n}\right)$ for all $\tau \in\left[\left(2^{p+1}-1\right) \theta, 2^{p+1} \theta\right]$. 
Returning to the original variables we see that

$$
\underset{K_{1}^{0}}{\operatorname{essinf}} u(x, t) \geq \mu:=\frac{1}{2} \theta^{\frac{1}{2-p}} \frac{\mu_{o}}{2} \exp \left[\frac{2^{2 p-1} \theta}{(2-p) \nu\left(\eta_{o} / 2\right)^{p}}\right]
$$

for all $t \in\left[\gamma_{1}, \gamma_{2}\right]$ where we have set

$$
\begin{gathered}
\gamma_{1}=\nu \mu_{o}^{2-p}\left(\frac{\eta_{o}}{2}\right)^{p}\left[\exp \left(\frac{2^{p-2}\left(2^{p+1}-1\right) \theta}{\nu\left(\eta_{o} / 2\right)^{p}}\right)-1\right], \\
\gamma_{2}=\nu \mu_{o}^{2-p}\left(\frac{\eta_{o}}{2}\right)^{p}\left[\exp \left(\frac{2^{2 p-1} \theta}{\nu\left(\eta_{o} / 2\right)^{p}}\right)-1\right] .
\end{gathered}
$$

Remark. One can see that the constant $\mu$, and consequently, the Hölder constants $\alpha_{1}$ and $\alpha_{2}$ deteriorate as $p \rightarrow 2$. Indeed it can be shown that these constants can be stabilized. One only needs to repeat the argument of Lemma 7.1 of [5] with obvious modifications.

\section{References}

[1] Y.Z. Chen and E. DiBenedetto, On the local behaviour of solutions of singular parabolic equations, Arch. Rational Mech. Anal. 103, no.4 (1988), 319-345.

[2] Y.Z. Chen and E. DiBenedetto, Boundary estimates for solutions of nonlinear degenerate parabolic systems, J. Reine Angew. Math. 395 (1989), 102-131.

[3] E. DiBenedetto, Degenerate parabolic equations, Universitext, Springer-Verlag, New York, 1993.

[4] E. DiBenedetto, U. Gianazza, V. Vespri, Local clustering of the non-zero set of functions in $W^{1,1}(E)$, Atti Accad. Naz. Lincei Cl. Sci. Fis. Mat. Natur. Rend. Lincei Mat. Appl., 17 (2006), no. 3, 223-225.

[5] E. DiBenedetto, U. Gianazza, V. Vespri, Harnack estimates for quasi-linear degenerate parabolic differential equations, Acta Math. 200 (2008), no. 2, 181-209.

[6] E. DiBenedetto, U. Gianazza, V. Vespri, A Geometric Approach to the Hölder Continuity of Solutions to Certain Singular Parabolic Partial Differential Equations, in preparation.

[7] E. DiBenedetto, J.M. Urbano, V. Vespri, Current issues on singular and degenerate evolution equations, Evolutionary equations, Vol. I, 169-286, Handb. Differ. Equ., North-Holland, Amsterdam, 2004.

[8] E. DiBenedetto, On the local behaviour of solutions of degenerate parabolic equations with measurable coefficients, Ann. Sc. Norm. Sup. Pisa Cl. Sc. Serie IV, XIII 3 (1986), 487-535.

[9] J.M. Urbano, The method of intrinsic scaling, Lecture Notes in Mathematics, 1930, Springer-Verlag, Berlin, 2008.

[10] J. L. Vazquez, The Porous Medium Equation: Mathematical Theory, Clarendon Press, Oxford Mathematical Monographs, 2006. 